\title{
Regularity of Hölder continuous solutions of the supercritical porous media equation
}

\author{
Wenxin $\mathrm{Yu}^{1 *}$, Yigang $\mathrm{He}^{2}$, Yaonan Tong ${ }^{1}$, Qiwu Luo ${ }^{1}$ and Xianming $\mathrm{Wu}^{1}$
}

${ }^{*}$ Correspondence:

slowbird@sohu.com

${ }^{1}$ College of Electrical and

Information Engineering, Hunan

University, Changsha, Hunan

410082, P.R. China

Full list of author information is

available at the end of the article

\begin{abstract}
In this paper, we present a regularity result for weak solutions of the $N$-dimensional ( $N=2$ or 3 ) porous media equation with supercritical $(\alpha<1)$ dissipation $\Lambda^{\alpha}$. If a Leray-Hopf weak solution is Hölder continuous $\theta \in C^{\delta}\left(R^{N}\right)$ with $\delta>1-\alpha$ on the time interval $[0, t]$, then it is actually a classical solution on $(0, t]$.
\end{abstract}

MSC: $76505 ; 76 \mathrm{D} 03$

Keywords: porous media equation; supercritical dissipation; regularity; weak solutions

\section{Introduction}

We use Darcy's law to describe the flow velocity, which reads

$$
v=-k(\nabla p+g \gamma \theta)
$$

where $v \in \mathbb{R}^{N}$ is the liquid discharge, $p$ is the scalar pressure, $\theta$ is the liquid temperature, $k$ is the matrix position-independent medium permeabilities in the different directions, respectively, divided by the viscosity, $g$ is the acceleration due to gravity and $\gamma \in \mathbb{R}^{N}$ is the last canonical vector $e_{N}$. For brevity, we only consider $k=g=1$.

In this article, we study the system of heat transfer with a fractional diffusion in an incompressible $N$ (2 or 3)-dimensional flow [1]

$$
(D P M)_{\alpha} \quad\left\{\begin{array}{l}
\partial_{t} \theta+v \cdot \nabla \theta+v \Lambda^{\alpha} \theta=0 \\
v=-(\nabla p+\gamma \theta), \quad \operatorname{div} v=0 \\
\theta(0, x)=\theta^{0}(x)
\end{array}\right.
$$

where $v>0$ is the dissipative coefficient, and the differential operator $\Lambda^{\alpha}$ is given by $\Lambda^{\alpha}:=(-\Delta)^{\frac{\alpha}{2}}$. Considering the scaling transform $\theta(t, x) \rightarrow \theta_{\lambda}(t, x):=\lambda^{\alpha-1} \theta\left(\lambda^{\alpha} t, \lambda x\right)$ for $\lambda>0$, the system will be divided into three cases: the case $\alpha=1$ is called the critical case, the case $\alpha>1$ is subcritical and the case $\alpha<1$ is supercritical.

Next, by rewriting Darcy's law, we obtain the expression of velocity $v$ only in terms of temperature $\theta[2,3]$. In the 2D case, thanks to the incompressibility, taking the curl operator first and the $\nabla^{\perp}:=\left(-\partial_{x_{2}}, \partial_{x_{1}}\right)$ operator second on both sides of Darcy's law, we have

$$
-\Delta v=\nabla^{\perp}\left(\partial_{x_{1}} \theta\right)=\left(-\partial_{x_{1}} \partial_{x_{2}} \theta, \partial_{x_{1}}^{2} \theta\right)
$$


thus the velocity $v$ can be recovered as

$$
v(t, x)=-\frac{1}{2 \pi} \int_{\mathbb{R}^{2}} \ln |x-y|\left(-\frac{\partial^{2} \theta}{\partial y_{2} \partial y_{1}}(t, y), \frac{\partial^{2} \theta}{\partial^{2} y_{1}}(t, y)\right) d y, \quad x \in \mathbb{R}^{2} .
$$

Through integration by parts, we finally get

$$
v(t, x)=-\frac{1}{2}(0, \theta(t, x))+\frac{1}{2 \pi} P V \int_{\mathbb{R}^{2}} H(x-y) \theta(t, y) d y, \quad x \in \mathbb{R}^{2},
$$

where the kernel $H(\cdot)$ is defined by

$$
H(x)=\left(\frac{2 x_{1} x_{2}}{|x|^{4}}, \frac{x_{2}^{2}-x_{1}^{2}}{|x|^{4}}\right) .
$$

Similarly, in the 3D case, applying the curl operator twice to Darcy's law, we get

$$
-\Delta v=\left(-\partial_{1} \partial_{3} \theta,-\partial_{2} \partial_{3} \theta, \partial_{1}^{2} \theta+\partial_{2}^{2} \theta\right)
$$

where $\partial_{i}:=\frac{\partial}{\partial x_{i}}$, thus

$$
v(t, x)=-\frac{2}{3}(0,0, \theta(t, x))+\frac{1}{4 \pi} P V \int_{\mathbb{R}^{3}} K(x-y) \theta(t, y) d y, \quad x \in \mathbb{R}^{3},
$$

where

$$
K(x)=\left(\frac{3 x_{1} x_{3}}{|x|^{5}}, \frac{3 x_{2} x_{3}}{|x|^{5}}, \frac{2 x_{3}^{2}-x_{1}^{2}-x_{2}^{2}}{|x|^{5}}\right) .
$$

We observe that, in general, each coefficient of $v(\cdot, t)$ (with $t$ as parameter) is only the linear combination of the Calderón-Zygmund singular integral (for the definition, see the sequel) of $\theta$ and $\theta$ itself. We write the general version as

$$
v:=\mathcal{T}(\theta)=\mathcal{C}(\theta)+\mathcal{S}(\theta),
$$

where $\mathcal{T}=\left(\mathcal{T}_{k}\right), \mathcal{C}=\left(\mathcal{C}_{k}\right), \mathcal{S}=\left(\mathcal{S}_{k}\right), 1 \leq k \leq N$, are all operators mapping scalar functions to vector-valued functions and $\mathcal{C}_{k}$ equals a constant multiplication operator, whereas $\mathcal{S}_{k}$ means a Calderón-Zygmund singular integral operator. Especially the corresponding specific forms in $2 \mathrm{D}$ or $3 \mathrm{D}$ are shown as (2) or (3).

We observe that the system $\left(D P M_{\alpha}\right)$ is not more than a dissipative transport diffusion equation with non-local divergence-free velocity field (the specific relationship between velocity and temperature as (4) shows). It shares many similarities with another flow model - 2D dissipative quasi-geostrophic (QG) equation, which has been intensively studied by many authors [4-11]. From a mathematical point of view, the system $\left(D P M_{\alpha}\right)$ is somewhat a generalization of $(\mathrm{QG})$ equation. Very recently, the system $\left(D P M_{\alpha}\right)$ was introduced and investigated by Córdoba and his group. In [2], the authors obtained some results on strong solutions, weak solutions and attractors for the dissipative system $\left(D P M_{\alpha}\right)$. For finite energy, they obtained global existence and uniqueness in the subcritical and critical cases. In the supercritical case, they obtained local results in $H^{s}, s>(N-\alpha) / 2+1$ and extended 
to be global under a small condition $\left\|\theta^{0}\right\|_{H^{s}} \leq c v$, for $s>N / 2+1$, where $c$ is a small fixed constant. In [3], they treated the nondissipative $(v=0) 2 \mathrm{D}$ case and obtained the local existence and uniqueness in the Hölder space $C^{\delta}$ for $0<\delta<1$ by the particle-trajectory method and gave some blowup criteria of smooth solutions.

In this paper we present a regularity result of weak solutions of the porous media equation with $\alpha<1$ (the supercritical case). The result asserts that if a Leray-Hopf weak solution $\theta$ of (1) is in the Hölder class $C^{\delta}$ with $\delta>1-\alpha$ on the time interval $[0, t]$, then it is actually a classical solution on $(0, t]$. The proof involves representing the functions in the Hölder space in terms of the Littlewood-Paley decomposition and using Besov space techniques. When $\theta$ is in $C^{\delta}$, it also belongs to the Besov space $\dot{B}_{p, \infty}^{\delta(1-2 / p)}$ for any $p \geq 2$. By taking $p$ sufficiently large, we have $\theta \in C^{\delta_{1}} \cap \dot{B}_{p, \infty}^{\delta_{1}}$ for $\delta_{1}>1-\alpha$. The idea is to show that $\theta \in C^{\delta_{2}} \cap \dot{B}_{p, \infty}^{\delta_{2}}$ with $\delta_{2}>\delta_{1}$. Through iteration, we establish that $\theta \in C^{\gamma}$ with $\gamma>1$. Then $\theta$ becomes a classical solution.

The rest of this paper is divided into two sections. Section 2 provides the definition of Besov spaces and necessary tools. Section 3 states and proves the main result.

Notation Throughout the paper, $C$ denotes various 'harmless' large finite constants, and $c$ denotes various 'harmless' small constants. We shall sometimes use $X \lesssim Y$ to denote the estimate $X \leq C Y$ for some $C$.

\section{Besov spaces and related tools}

In this preparatory section, we give the definition of Besov spaces based on the LittlewoodPaley decomposition, introduce the Calderón-Zygmund singular integral, and finally we review some important results that will be used in the following.

Let us recall the Littlewood-Paley decomposition. Let $\mathcal{S}\left(\mathbb{R}^{N}\right)$ be the Schwartz class of rapidly decreasing functions. Given $f \in \mathcal{S}\left(\mathbb{R}^{N}\right)$, its Fourier transform $\mathcal{F} f=\hat{f}$ is defined by

$$
\hat{f}(\xi)=(2 \pi)^{-\frac{N}{2}} \int_{\mathbb{R}^{N}} e^{-i x \cdot \xi} f(x) d x
$$

Choose two nonnegative radial functions $\chi, \varphi \in \mathcal{S}\left(\mathbb{R}^{N}\right)$, supported respectively in $\mathcal{B}=\{\xi \in$ $\left.\mathbb{R}^{N},|\xi| \leq \frac{4}{3}\right\}$ and $\mathcal{C}=\left\{\xi \in \mathbb{R}^{N}, \frac{3}{4} \leq|\xi| \leq \frac{8}{3}\right\}$, such that

$$
\begin{aligned}
& \chi(\xi)+\sum_{j \geq 0} \varphi\left(2^{-j} \xi\right)=1, \quad \xi \in \mathbb{R}^{N}, \\
& \sum_{j \in Z} \varphi\left(2^{-j} \xi\right)=1, \quad \xi \in \mathbb{R}^{N} \backslash\{0\} .
\end{aligned}
$$

Setting $\varphi_{j}(\xi)=\varphi\left(2^{-j} \xi\right)$. Let $h=\mathcal{F}^{-1} \varphi$ and $\tilde{h}=\mathcal{F}^{-1} \chi$, we define the frequency localization operator as follows:

$$
\begin{aligned}
& \Delta_{j} f=\varphi\left(2^{-j} D\right) f=2^{j N} \int_{R^{N}} h\left(2^{j} y\right) f(x-y) d y \\
& S_{j} f=\sum_{k \leq j-1} \Delta_{k} f=\chi\left(2^{-j} D\right) f=2^{j N} \int_{R^{N}} \tilde{h}\left(2^{j} y\right) f(x-y) d y .
\end{aligned}
$$


Informally, $\Delta_{j}=S_{j}-S_{j-1}$ is a frequency projection to the annulus $\left\{|\xi| \approx 2^{j}\right\}$, while $S_{j}$ is a frequency projection to the ball $\left\{|\xi| \lesssim 2^{j}\right\}$. One easily verifies that with our choice of $\varphi$,

$$
\Delta_{j} \Delta_{k} f \equiv 0 \quad \text { if }|j-k| \geq 2 \quad \text { and } \quad \Delta_{j}\left(S_{k-1} f \Delta_{k} f\right) \equiv 0 \quad \text { if }|j-k| \geq 5 .
$$

Now we give the definitions of Besov spaces.

Definition 2.1 Let $s \in \mathbb{R}, 1 \leq p, q \leq \infty$, the homogeneous Besov space $\dot{B}_{p, q}^{s}$ is defined by

$$
\dot{B}_{p, q}^{s}=\left\{f \in \mathcal{Z}^{\prime}\left(\mathbb{R}^{N}\right) ;\|f\|_{\dot{B}_{p, q}^{s}}<\infty\right\}
$$

Here,

$$
\|f\|_{\dot{B}_{p, q}^{s}}= \begin{cases}\left(\sum_{j \in \mathbb{Z}} 2^{j s q}\left\|\Delta_{j} f\right\|_{p}^{q}\right)^{\frac{1}{q}} & \text { for } q<\infty \\ \sup _{j \in \mathbb{Z}}\left\|\Delta_{j} f\right\|_{p} & \text { for } q=\infty\end{cases}
$$

and $\mathcal{Z}^{\prime}\left(\mathbb{R}^{N}\right)$ denotes the dual space of $\mathcal{Z}^{\prime}\left(\mathbb{R}^{N}\right)=\left\{f \in \mathcal{S}\left(\mathbb{R}^{N}\right) ; \partial^{\gamma} \hat{f}(0)=0 ; \forall \gamma \in \mathbb{N}^{N}\right.$ multiindex $\}$ and can be identified by the quotient space of $\mathcal{S}^{\prime} / \mathcal{P}$ with the polynomials space $\mathcal{P}$.

The following proposition lists a few simple facts that we will use in the subsequent section. The proof is rather standard and one can refer to [12].

Proposition 2.2 Assume that $s \in \mathbb{R}$ and $p, q \in[1, \infty]$.

(1) If $1 \leq q_{1} \leq q_{2} \leq \infty$, then $\dot{B}_{p, q_{1}}^{s} \subset \dot{B}_{p, q_{2}}^{s}$.

(2) (Besov embedding) If $1 \leq p_{1} \leq p_{2} \leq \infty$ and $s_{1}=s_{2}+n\left(\frac{1}{p_{1}}-\frac{1}{p_{2}}\right)$, then $\dot{B}_{p_{1}, q}^{s_{1}}\left(\mathbb{R}^{N}\right) \subset \dot{B}_{p_{2}, q}^{s_{2}}\left(\mathbb{R}^{N}\right)$.

(3) If $1<p<\infty$, then

$$
\dot{B}_{p, \min (p, 2)}^{s} \subset \dot{W}^{s, p} \subset \dot{B}_{p, \max (p, 2)}^{s},
$$

where $\dot{W}^{s, p}$ denotes a standard homogeneous Sobolev space.

We next introduce the classical Bernstein inequality [13]

Lemma 2.3 Let $\mathcal{B}$ be a ball, $\mathcal{C}$ be a ring, $0 \leq a \leq b \leq \infty$. Then $\forall k \in \mathbb{Z}^{+} \cup\{0\}, \forall \lambda>0$, there exists a constant $C>0$ such that

$$
\begin{aligned}
& \sup _{|\alpha|=k}\left\|\partial^{\alpha} f\right\|_{L^{b}} \leq C \lambda^{k+N\left(\frac{1}{a}-\frac{1}{b}\right)}\|f\|_{L^{a}} \quad \text { if } \operatorname{supp} \mathcal{F} f \subset \lambda \mathcal{B}, \\
& C^{-1} \lambda^{k}\|f\|_{L^{a}} \leq \sup _{|\alpha|=k}\left\|\partial^{\alpha} f\right\|_{L^{a}} \leq C \lambda^{k}\|f\|_{L^{a}} \quad \text { if } \operatorname{supp} \mathcal{F} f \subset \lambda \mathcal{C} .
\end{aligned}
$$

Similar inequalities hold for the fractional derivative $\Lambda^{\beta}$.

The following proposition provides a lower bound for an integral that originates from the dissipative term in the process of $L^{p}$ estimates (see $\left.[6,14]\right)$. 
Proposition 2.4 Assume either $\alpha \geq 0$ and $p=2$ or $0 \leq \alpha \leq 1$ and $2<p<\infty$. Let $j$ be an integer and $f \in \mathcal{S}^{\prime}$. Then

$$
\int_{\mathbb{R}^{N}}\left|\Delta_{j} f\right|^{p-2} \Delta_{j} f \Lambda^{2 \alpha} \Delta_{j} f d x \geq C 2^{2 \alpha j}\left\|\Delta_{j} f\right\|_{L^{p}}^{p}
$$

for some constant $C$ depending on $N, \alpha$ and $p$.

The classical Calderón-Zygmund singular integrals are operators of the form

$$
T_{c z} f(x):=P V \int_{\mathbb{R}^{N}} \frac{\Omega\left(y^{\prime}\right)}{|y|^{N}} f(x-y) d y=\lim _{\epsilon \rightarrow 0} \int_{|y|>\epsilon} \frac{\Omega\left(y^{\prime}\right)}{|y|^{N}} f(x-y) d y,
$$

where $\Omega$ is defined on the unit sphere of $\mathbb{R}^{N}, \mathbb{S}^{N-1}$, and is integrable with zero average, and where $y^{\prime}:=\frac{y}{|y|} \in \mathcal{S}^{N-1}$. Clearly, the definition is meaningful for Schwartz functions. Moreover, if $\Omega \in C^{1}\left(\mathcal{S}^{N-1}\right), T_{c z}$ is $L^{p}$ bounded, $1<p<\infty$.

The general version (4) of the relationship between $v$ and $\theta$ is in fact ensured by the following result (see, e.g., [15]).

Lemma 2.5 Let $m \in C^{\infty}\left(\mathbb{R}^{N} \backslash\{0\}\right)$ be a homogeneous function of degree 0 , and let $T_{m}$ be the corresponding multiplier operator defined by $\left(T_{m} f\right)^{\wedge}=m \hat{f}$, then there exist $a \in \mathbb{C}$ and $\Omega \in C^{\infty}\left(\mathbb{S}^{N-1}\right)$ with zero average such that for any Schwartz function $f$,

$$
T_{m} f=a f+P V \frac{\Omega\left(x^{\prime}\right)}{|x|^{N}} * f .
$$

Remark 2.1 Since $-\Delta v=\left(\partial_{1} \partial_{N} \theta, \ldots,-\partial_{N-1} \partial_{N} \theta, \partial_{1}^{2} \theta+\cdots+\partial_{N-1}^{2} \theta\right)$, the Fourier multiplier of the operator $\mathcal{T}$ is rather clear. In fact, each component of its multiplier is the linear combination of the term like $\frac{\xi_{i} \xi_{j}}{|\xi|^{2}}, i, j \in\{1,2, \ldots, N\}$, which of course belongs to $C^{\infty}\left(\mathbb{R}^{N} \backslash\{0\}\right)$ and is homogeneous of degree 0 .

\section{The main theorem and its proof}

Theorem 3.1 Let $\theta$ be a Leray-Hopf weak solution of (1), namely

$$
\theta \in L^{\infty}\left([0, \infty) ; L^{2}\left(\mathbb{R}^{N}\right)\right) \cap L^{2}\left([0, \infty) ; \dot{H}^{\alpha / 2}\left(\mathbb{R}^{N}\right)\right)
$$

Let $\delta>1-\alpha$ and let $0<t<\infty$. If

$$
\theta \in L^{\infty}\left([0, t] ; C^{\delta}\left(\mathbb{R}^{N}\right)\right)
$$

then $\theta \in C^{\infty}\left((0, t] \times \mathbb{R}^{N}\right)$ for $N=2$ or 3 .

Proof First we notice that (5) and (6) imply that

$$
\theta \in L^{\infty}\left([0, t] ; \dot{B}_{p, \infty}^{\delta_{1}}\left(\mathbb{R}^{N}\right)\right)
$$

for any $p \geq 2$ and $\delta_{1}=\delta\left(1-\frac{N}{p}\right)$. In fact, for any $\tau \in[0, t]$,

$$
\|\theta(\cdot, \tau)\|_{\dot{B}_{p, \infty}^{\delta_{1}}}=\sup _{j} 2^{\delta_{1} j}\left\|\Delta_{j} \theta\right\|_{L^{p}} \leq \sup _{j} 2^{\delta_{1} j}\left\|\Delta_{j} \theta\right\|_{L^{\infty}}^{1-\frac{2}{p}}\left\|\Delta_{j} \theta\right\|_{L^{2}}^{\frac{2}{p}} \leq\|\theta(\cdot, \tau)\|_{C^{\delta}}^{1-\frac{2}{p}}\|\theta(\cdot, \tau)\|_{L^{2}}^{\frac{2}{p}} .
$$


Since $\delta>1-\alpha$, we have $\delta_{1}>1-\alpha$ when

$$
p>p_{0} \equiv \frac{N \delta}{\delta-(1-\alpha)}
$$

Next, we show that

$$
\theta \in L^{\infty}\left([0, t] ; \dot{B}_{p, \infty}^{\delta_{1}} \cap C^{\delta_{1}}\right)
$$

implies

$$
\theta(\cdot, t) \in \dot{B}_{p, \infty}^{\delta_{2}} \cap C^{\delta_{2}}
$$

for some $\delta_{2}>\delta_{1}$ to be specified. Let $j$ be an integer. Applying $\Delta_{j}$ to the first equation of (1), we get

$$
\partial_{t} \Delta_{j} \theta+v \Lambda^{\alpha} \Delta_{j} \theta=-\Delta_{j}(v \cdot \nabla \theta)
$$

By Bony's notion of paraproduct,

$$
\Delta_{j}(v \cdot \nabla \theta)=\sum_{|k-j| \leq 4} \Delta_{j}\left(S_{k-1} v \cdot \nabla \Delta_{k} \theta\right)+\sum_{|k-j| \leq 4} \Delta_{j}\left(\Delta_{k} v \cdot \nabla S_{k-1} \theta\right) \sum_{\substack{k \geq j-2 \\|k-l| \leq 1}} \Delta_{j}\left(\Delta_{k} v \cdot \nabla \Delta_{l} \theta\right) .
$$

Multiplying (7) by $p\left|\Delta_{j} \theta\right|^{p-2} \Delta_{j} \theta$, integrating with respect to $x$ and applying the lower bound

$$
\int_{\mathbb{R}^{N}}\left|\Delta_{j} f\right|^{p-2} \Delta_{j} f \Lambda^{2 \alpha} \Delta_{j} f d x \geq C 2^{2 \alpha j}\left\|\Delta_{j} f\right\|_{L^{p}}^{p}
$$

of Proposition 2.4, we obtain

$$
\begin{aligned}
\frac{d}{d t}\left\|\Delta_{j} \theta\right\|_{L^{p}}^{p}+C \nu 2^{\alpha j}\left\|\Delta_{j} \theta\right\|_{L^{p}}^{p} \leq & -p \sum_{|k-j| \leq 4} \int_{\mathbb{R}^{N}}\left|\Delta_{j} \theta\right|^{p-2} \Delta_{j} \theta \Delta_{j}\left(S_{k-1} v \cdot \nabla \Delta_{k} \theta\right) d x \\
& -p \sum_{|k-j| \leq 4} \int_{\mathbb{R}^{N}}\left|\Delta_{j} \theta\right|^{p-2} \Delta_{j} \theta \Delta_{j}\left(\Delta_{k} v \cdot \nabla S_{k-1} \theta\right) d x \\
& -p \sum_{\substack{k \geq j-2 \\
|k-l| \leq 1}} \int_{\mathbb{R}^{N}}\left|\Delta_{j} \theta\right|^{p-2} \Delta_{j} \theta \Delta_{j}\left(\Delta_{l} v \cdot \nabla \Delta_{k} \theta\right) d x \\
= & I_{1}+I_{2}+I_{3} .
\end{aligned}
$$

We now estimate $I_{1}$. The standard idea is to decompose it into three terms: one with commutator, one that becomes zero due to the divergence-free condition and the rest. That is, we rewrite $I_{1}$ as

$$
\begin{aligned}
I_{1}= & -p \sum_{|k-j| \leq 4} \int_{\mathbb{R}^{N}}\left|\Delta_{j} \theta\right|^{p-2} \Delta_{j} \theta \cdot\left[\Delta_{j}, S_{k-1} v \cdot \nabla\right] \Delta_{k} \theta d x \\
& -p \int_{\mathbb{R}^{N}}\left|\Delta_{j} \theta\right|^{p-2} \Delta_{j} \theta \cdot\left(S_{j} v \cdot \nabla \Delta_{j} \theta\right) d x
\end{aligned}
$$




$$
\begin{aligned}
& -p \sum_{|k-j| \leq 1} \int_{\mathbb{R}^{N}}\left|\Delta_{j} \theta\right|^{p-2} \Delta_{j} \theta \cdot\left(S_{k-1} v-S_{j} v\right) \cdot \nabla \Delta_{j} \Delta_{k} \theta d x \\
= & I_{11}+I_{12}+I_{13},
\end{aligned}
$$

where we have used the simple fact that $\sum_{|k-j| \leq 1} \Delta_{k} \Delta_{j}=\Delta_{j}$, and the brackets [ ] represent the commutator, namely

$$
\left[\Delta_{j}, S_{k-1} v \cdot \nabla\right] \Delta_{k} \theta=\Delta_{j}\left(S_{k-1} v \cdot \nabla \Delta_{k} \theta\right)-S_{k-1} v \cdot \nabla \Delta_{j} \Delta_{k} \theta
$$

Since $u$ is divergence free, $I_{12}$ becomes zero. We now bound $I_{11}$ and $I_{13}$. By Hölder's inequality,

$$
I_{11} \leq p\left\|\Delta_{j} \theta\right\|_{L^{p}}^{p-1} \sum_{|k-j| \leq 4}\left\|\left[\Delta_{j}, S_{k-1} v \cdot \nabla\right] \Delta_{k} \theta\right\|_{L^{p}} .
$$

To bound the commutator, we have, by the definition of $\Delta_{j}$,

$$
\left[\Delta_{j}, S_{k-1} v \cdot \nabla\right] \Delta_{k} \theta=2^{k N} \int_{\mathbb{R}^{N}} h\left(2^{k}(x-y)\right)\left(S_{k-1}(v)(x)-S_{k-1}(v)(y)\right) \cdot \nabla \Delta_{k} \theta d y .
$$

Using the fact that $\theta \in C^{\delta_{1}}$ and thus

$$
\left\|S_{k-1}(v)(x)-S_{k-1}(v)(y)\right\|_{L^{\infty}} \leq\|v\|_{C^{\delta_{1}}}|x-y|^{\delta_{1}}
$$

we obtain

$$
\left\|\left[\Delta_{j}, S_{k-1} v \cdot \nabla\right] \Delta_{k} \theta\right\|_{L^{p}} \leq 2^{-\delta_{1} j}\|v\|_{C^{\delta_{1}}} 2^{k}\left\|\Delta_{k} \theta\right\|_{L^{p}}
$$

Therefore,

$$
\begin{aligned}
\left|I_{11}\right| & \leq C p\left\|\Delta_{j} \theta\right\|_{L^{p}}^{p-1} 2^{-\delta_{1} j}\|v\|_{C^{\delta_{1}}} \sum_{|k-j| \leq 4} 2^{k}\left\|\Delta_{k} \theta\right\|_{L^{p}} \\
& \leq C p\left\|\Delta_{j} \theta\right\|_{L^{p}}^{p-1} 2^{\left(1-2 \delta_{1}\right) j}\|v\|_{C^{\delta_{1}}} \sum_{|k-j| \leq 4} 2^{\delta_{1} k}\left\|\Delta_{k} \theta\right\|_{L^{p}} 2^{(k-j)\left(1-\delta_{1}\right)} \\
& \leq C p\left\|\Delta_{j} \theta\right\|_{L^{p}}^{p-1} 2^{\left(1-2 \delta_{1}\right) j}\|v\|_{C^{\delta_{1}}}\|\theta\|_{\dot{B}_{p, \infty}^{\delta_{1}}} .
\end{aligned}
$$

The estimate for $I_{13}$ is straightforward. By Hölder's inequality,

$$
\begin{aligned}
\left|I_{13}\right| & \leq C p\left\|\Delta_{j} \theta\right\|_{L^{p}}^{p-1} \sum_{|k-j| \leq 4}\left\|S_{k-1} v-S_{j} v\right\|_{L^{p}}\left\|\nabla \Delta_{j} \theta\right\|_{L^{\infty}} \\
& \leq C p\left\|\Delta_{j} \theta\right\|_{L^{p}}^{p-1} 2^{\left(1-\delta_{1}\right) j}\|\theta\|_{C^{\delta_{1}}} \sum_{|k-j| \leq 4}\left\|\Delta_{k} v\right\|_{L^{p}} \\
& \leq C p\left\|\Delta_{j} \theta\right\|_{L^{p}}^{p-1} 2^{\left(1-2 \delta_{1}\right) j}\|\theta\|_{C^{\delta_{1}}} \sum_{|k-j| \leq 4} 2^{\delta_{1} k}\left\|\Delta_{k} v\right\|_{L^{p}} 2^{(j-k) \delta_{1} k} \\
& \leq C p\left\|\Delta_{j} \theta\right\|_{L^{p}}^{p-1} 2^{\left(1-2 \delta_{1}\right) j}\|\theta\|_{C^{\delta_{1}}}\|v\|_{\dot{B}_{p, \infty}^{\delta_{1}}} .
\end{aligned}
$$


We then bound $I_{2}$. By Hölder's inequality, Bernstein's inequality and the fact $1-\delta_{1}>0$, we obtain

$$
\begin{aligned}
I_{2} & \leq C p\left\|\Delta_{j} \theta\right\|_{L^{p}}^{p-1} \sum_{|k-j| \leq 4}\left\|\Delta_{k} v\right\|_{L^{p}}\left\|\nabla S_{k-1} \theta\right\|_{L^{\infty}} \\
& \leq C p\left\|\Delta_{j} \theta\right\|_{L^{p}}^{p-1} \sum_{|k-j| \leq 4}\left\|\Delta_{k} v\right\|_{L^{p}} \sum_{k^{\prime} \leq k-2} 2^{k^{\prime}}\left\|\Delta_{k^{\prime}} \theta\right\|_{L^{\infty}} \\
& \leq C p\left\|\Delta_{j} \theta\right\|_{L^{p}}^{p-1} \sum_{|k-j| \leq 4}\left\|\Delta_{k} v\right\|_{L^{p}} 2^{\left(1-\delta_{1}\right) k} \sum_{k^{\prime} \leq k-2} 2^{\left(k^{\prime}-k\right)\left(1-\delta_{1}\right)} 2^{k^{\prime} \delta_{1}}\left\|\Delta_{k^{\prime}} \theta\right\|_{L^{\infty}} \\
& \leq C p\left\|\Delta_{j} \theta\right\|_{L^{p}}^{p-1}\|\theta\|_{C^{\delta_{1}}} \sum_{|k-j| \leq 4}\left\|\Delta_{k} v\right\|_{L^{p}} 2^{\left(1-\delta_{1}\right) k} \\
& \leq C p\left\|\Delta_{j} \theta\right\|_{L^{p}}^{p-1}\|\theta\|_{C^{\delta_{1}}} 2^{\left(1-2 \delta_{1}\right) j} \sum_{|k-j| \leq 4} 2^{\delta_{1} k}\left\|\Delta_{k} v\right\|_{L^{p}} 2^{(k-j)\left(1-2 \delta_{1}\right)} \\
& \leq C p\left\|\Delta_{j} \theta\right\|_{L^{p}}^{p-1}\|\theta\|_{C^{\delta_{1}}} 2^{\left(1-2 \delta_{1}\right) j}\|v\|_{\dot{B}_{p, \infty}^{\delta_{1}}} .
\end{aligned}
$$

Last, we bound $I_{3}$. By Hölder's inequality and Bernstein's inequality,

$$
\begin{aligned}
I_{3} & \leq C p\left\|\Delta_{j} \theta\right\|_{L^{p}}^{p-1}\left\|\Delta_{j} \nabla \cdot\left(\sum_{\substack{k \geq j-2 \\
|k-l| \leq 1}} \Delta_{l} v \Delta_{k} \theta\right)\right\|_{L^{p}} \\
& \leq C p\left\|\Delta_{j} \theta\right\|_{L^{p}}^{p-1} 2^{j}\|v\|_{C^{\delta_{1}}} \sum_{k \geq j-2} 2^{-\delta_{1} k}\left\|\Delta_{k} \theta\right\|_{L^{p}} \\
& \leq C p\left\|\Delta_{j} \theta\right\|_{L^{p}}^{p-1} 2^{\left(1-2 \delta_{1}\right) j}\|v\|_{C^{\delta_{1}}} \sum_{k \geq j-2} 2^{-2 \delta_{1}(k-j)} 2^{\delta_{1} k}\left\|\Delta_{k} \theta\right\|_{L^{p}} \\
& \leq C p\left\|\Delta_{j} \theta\right\|_{L^{p}}^{p-1} 2^{\left(1-2 \delta_{1}\right) j}\|v\|_{C^{\delta_{1}}}\|\theta\|_{\dot{B}_{p, \infty}^{\delta_{1}}} .
\end{aligned}
$$

Inserting the estimates for $I_{1}, I_{2}$ and $I_{3}$ in (8) and eliminating $p\left\|\Delta_{j} \theta\right\|_{L^{p}}^{p-1}$ from both sides, we get

$$
\begin{aligned}
\frac{d}{d t}\left\|\Delta_{j} \theta\right\|_{L^{p}}+C \nu 2^{\alpha j}\left\|\Delta_{j} \theta\right\|_{L^{p}} \leq & 2^{\left(1-2 \delta_{1}\right) j}\|v\|_{C^{\delta_{1}}}\|\theta\|_{\dot{B}_{p, \infty}^{\delta_{1}}}+2^{\left(1-2 \delta_{1}\right) j}\|\theta\|_{C^{\delta_{1}}}\|v\|_{\dot{B}_{p, \infty}^{\delta_{1}}} \\
& +2^{\left(1-2 \delta_{1}\right) j}\|\theta\|_{C^{\delta_{1}}}\|v\|_{\dot{B}_{p, \infty}^{\delta_{1}}}+2^{\left(1-2 \delta_{1}\right) j}\|v\|_{C^{\delta_{1}}}\|\theta\|_{\dot{B}_{p, \infty}^{\delta_{1}}} .
\end{aligned}
$$

Integrating with time $t$, we have

$$
\begin{aligned}
\left\|\Delta_{j} \theta(t)\right\|_{L^{p}} \leq & e^{-C \nu 2^{\alpha j} t}\left\|\Delta_{j} \theta(0)\right\|_{L^{p}} \\
& +C \int_{0}^{t} e^{-C \nu 2^{\alpha j}(t-\tau)} 2^{\left(1-2 \delta_{1}\right) j}\left(\|v\|_{C^{\delta_{1}}}\|\theta\|_{\dot{B}_{p, \infty}^{\delta_{1}}}+\|\theta\|_{C^{\delta_{1}}}\|v\|_{\dot{B}_{p, \infty}^{\delta_{1}}}\right) d \tau .
\end{aligned}
$$

Multiplying both sides by $2^{\left(\alpha+2 \delta_{1}-1\right) j}$ and taking the supremum with respect to $j$, we get

$$
\begin{aligned}
\|\theta(t)\|_{\dot{B}_{p, \infty}^{\alpha+2 \delta_{1}-1} \leq} & \sup _{j}\left\{e^{-C \nu 2^{\alpha j} t} 2^{\left(\alpha+\delta_{1}-1\right) j}\right\}\|\theta(0)\|_{\dot{B}_{p, \infty}^{\delta_{1}}} \\
& +C v^{-1} \sup _{j}\left\{\left(1-e^{-C \nu 2^{\alpha j} t}\right)\right\} \max _{\tau \in[0, t]}\|\theta(\tau)\|_{\dot{B}_{p, \infty}^{\delta_{1}}}\|\theta(\tau)\|_{C^{\delta_{1}}} .
\end{aligned}
$$


Here we have used the fact that

$$
\|v\|_{C^{\delta_{1}}} \leq\|\theta\|_{C^{\delta_{1}}} \quad \text { and } \quad\|v\|_{\dot{B}_{p, \infty}^{\delta_{1}}} \leq\|\theta\|_{\dot{B}_{p, \infty}^{\delta_{1}}} .
$$

Therefore, we conclude that if

$$
\theta \in L^{\infty}\left([0, t] ; \dot{B}_{p, \infty}^{\delta_{1}} \cap C^{\delta_{1}}\right)
$$

then

$$
\theta(\cdot, t) \in \dot{B}_{p, \infty}^{\alpha+2 \delta_{1}-1}
$$

Since $\delta_{1}>1-\alpha$, we have $2 \delta_{1}+\alpha-1>\delta_{1}$ and thus gain regularity. In addition, according to the Besov embedding of Proposition 2.2,

$$
\dot{B}_{p, \infty}^{\alpha+2 \delta_{1}-1} \subset \dot{B}_{\infty, \infty}^{\delta_{2}}
$$

where

$$
\delta_{2}=2 \delta_{1}+\alpha-1-\frac{N}{p}=\delta_{1}+\left(\delta_{1}-\left(1-\alpha+\frac{N}{p}\right)\right) .
$$

We have $\delta_{2}>\delta_{1}$ when

$$
p>p_{1} \equiv \frac{N}{\delta_{1}-(1-\alpha)} .
$$

Noticing that

$$
\dot{B}_{\infty, \infty}^{\delta_{2}} \cap L^{\infty}=C^{\delta_{2}},
$$

we conclude that, for $p>\max \left\{p_{0}, p_{1}\right\}$,

$$
\theta(\cdot, t) \in \dot{B}_{p, \infty}^{\delta_{2}} \cap C^{\delta_{2}}
$$

for some $\delta_{2}>\delta_{1}$. The above process can then be iterated with $\delta_{1}$ replaced by $\delta_{2}$. A finite number of iterations allow us to obtain that

$$
\theta(\cdot, t) \in C^{\gamma}
$$

for some $\gamma>1$. The regularity in the spatial variable can then be converted into regularity in time. We have thus established that $\theta$ is a classical solution to the supercritical porous media equation. Higher regularity can be proved by well-known methods. 
Authors' contributions

In this paper, WY carried 'Regularity of Hoder continuous solutions of the supercritical porous media equation'. YH, YT, QL and XW participated in the analysis. All authors read and approved the final manuscript.

\section{Author details}

${ }^{1}$ College of Electrical and Information Engineering, Hunan University, Changsha, Hunan 410082, P.R. China. ${ }^{2}$ School of Electrical and Automation Engineering, Hefei University of Technology, Hefei, Anhui 230009, P.R. China.

\section{Acknowledgements}

This work was supported by the National Natural Science Funds of China for Distinguished Young Scholar under Grant No. 50925727, the National Defense Advanced Research Project Grant Nos. C1120110004, 9140A27020211DZ5102, the Key Grant Project of Chinese Ministry of Education under Grant No. 313018, and the Fundamental Research Funds for the Central Universities (2012HGCX0003).

Received: 25 July 2013 Accepted: 27 August 2013 Published: 07 Nov 2013

\section{References}

1. Nield, DA, Bejan, A: Convection in Porous Media. Springer, New York (1999)

2. Castro, A, Córdoba, D, Gancedo, F, Orive, R: Incompressible flow in porous media with fractional diffusion. Nonlinearity 22, 1791-1815 (2009)

3. Córdoba, D, Gancedo, F, Orive, R: Analytical behaviour of the two-dimensional incompressible flow in porous media. J. Math. Phys. 48, 1-19 (2007)

4. Abidi, H, Hmidi, T: On the global well posedness of the critical quasi-geostrophic equation. SIAM J. Math. Anal. 40 167-185 (2008)

5. Caffarelli, L, Vasseur, V: Drift diffusion equations with fractional diffusion and the quasi-geostrophic equations. Ann. Math. 171, 1903-1930 (2010)

6. Chen, Q, Miao, C, Zhang, Z: A new Bernstein's inequality and the 2D dissipative quasigeostrophic equation. Commun. Math. Phys. 271, 821-838 (2007)

7. Constantin, $\mathrm{P}, \mathrm{Wu}$, J: Regularity of Hölder continuous solutions of the supercritical quasi-geostrophic equation. Ann Inst. Henri Poincaré, Anal. Non Linéaire 25, 1103-1110 (2008)

8. Constantin, P, Iyer, G, Wu, J: Global regularity for a modified critical dissipative quasigeostrophic equation. Indiana Univ. Math. J. 57, 2681-2692 (2008)

9. Córdoba, A, Córdoba, D: A maximum principle applied to the quasi-geostrophic equations. Commun. Math. Phys. 249, 511-528 (2004)

10. Hmidi, T, Kernaani, S: Global solutions of the supercritical 2D dissipative quasigeostrophic equation. Adv. Math. 214, 618-638 (2007)

11. Kislev, A, Nazarov, F, Volberg, A: Global well-posedness for the critical 2D dissipative quasi-geostrophic equation. Invent. Math. 167, 445-453 (2007)

12. Triebel, $\mathrm{H}$ : Theory of Function Spaces. Monograph in Mathematics, vol. 78. Birkhäuser, Basel (1983)

13. Chemin, J-Y: Perfect Incompressible Fluids. Clarendon, Oxford (1998)

14. Wu, J: The quasi-geostrophic equation and its two regularizations. Commun. Partial Differ. Equ. 27, 1161-1181 (2002)

15. Duoandikoetxea, J: Fourier Analysis (Translated and revised by D. Cruz-Uribe). GSM, vol. 29. Am. Math. Soc, Providence (2001)

10.1186/1687-2770-2013-225

Cite this article as: Yu et al.: Regularity of Hölder continuous solutions of the supercritical porous media equation. Boundary Value Problems 2013, 2013:225

\section{Submit your manuscript to a SpringerOpen ${ }^{\circ}$ journal and benefit from:}

- Convenient online submission

- Rigorous peer review

- Immediate publication on acceptance

- Open access: articles freely available online

- High visibility within the field

- Retaining the copyright to your article 